\title{
Neural Networks and Its Application in Engineering
}

\author{
Oludele Awodele and Olawale Jegede \\ Dept. of Computer Science and Mathematics, \\ Babcock University, Nigeria
}

\author{
delealways@yahoo.com; jegede1@yahoo.com
}

\begin{abstract}
Neural Network (NN) has emerged over the years and has made remarkable contribution to the advancement of various fields of endeavor. The purpose of this work is to examine neural networks and their emerging applications in the field of engineering, focusing more on Controls. In this work, we have examined the various architectures of $\mathrm{NN}$ and the learning process. The needs for neural networks, training of neural networks, and important algorithms used in realizing neural networks have also been briefly discussed. Neural network application in control engineering has been extensively discussed, whereas its applications in electrical, civil and agricultural engineering were also examined. We concluded by identifying limitations, recent advances and promis ing future research directions.
\end{abstract}

Keywords. Neural Network, Artificial Neural Network

\section{Introduction}

Whenever we talk about a neural network, we should more properly say "artific ial neural network" (ANN), because that is what we mean most of the time. Artificial ne ural networks are computers whose architecture is modeled after the brain. They typically cons ist of many hundreds of simple processing units which are wired together in a complex communication network. Each unit or node is a simplified model of a real neuron which fires (sends off a new signal) if it receives a sufficiently strong input signal from the other nodes to which it is connected.

An Artificial Neural Network (ANN) is an information processing paradigm that is inspired by the way biological nervous systems, such as the brain, process information. The key element of this paradigm is the novel structure of the information processing system. It is composed of a large number of highly interconnected processing elements (neurons) work ing in unison to solve specific problems. ANNs, like people, learn by example. According to Michael Mozer of the University of Colorado, "The neural network is structured to performnonlinear Bayesian classification".

Material published as part of this publication, either on-line or in print, is copy righted by the Informing Science Institute. Permission to make digital or paper copy of part or all of these works for personal or classroom use is granted without fee provided that the copies are not made or distributed for profit or commercial advantage AND that copies 1) bear this notice in full and 2) give the full citation on the first page. It is permissible to abstract these works so long as credit is given. To copy in all other cases or to republish or to post on a server or to redistribute to lists requires specific permission and payment of a fee. Contact Publisher@InformingScience.org to request redistribution permission.
A neural network could be also be described as a system composed of many simple processing elements operating in parallel whose function is determined by network structure, connection strengths, and the processing performed at computing elements or nodes (DARPA Neural Network Study, 1988).

It resembles the brain in two respects: 
1. Knowledge is acquired by the network through a learning process.

2. Interneuron connection strengths known as synaptic weights are used to store the knowledge (Haykin, 1999).

\section{Historical Background}

The history of neural networks can be divided into several periods: from when developed models of neural networks based on their understanding of neurology, to when neuroscience became influential in the development of neural networks. Psychologists and engineers also contributed to the progress of neural network simulations. Neurally based chips are emerging and applications to complex problems developing. Clearly, today is a period of transition for neural network technology.

\section{Why use neural networks?}

Neural networks, with their remarkable ability to derive meaning from complicated or imprecise data, can be used to extract patterns and detect trends that are too complex to be noticed by either humans or other computer techniques. A trained neural network can be thought of as an "expert" in the category of information it has been given to analyze. This expert can then be used to provide projections given new situations of interest and answer "what if" questions. Other advantages include: Adaptive learning, Self-Organization, Real Time Operation and Fault Tolerance via Redundant Information Coding

Neural networks process information in a similar way the human brain does. The network is composed of a large number of highly interconnected processing elements (neurones) working in parallel to solve a specific problem. Neural networks learn by example. They cannot be programmed to perform a specific task. The examples must be selected carefully otherwise useful time is wasted or even worse the network might be functioning incorrectly. The disadvantage is that because the network finds out how to solve the problem by itself, its operation can be unpredictable.

Neural networks and conventional algorithmic computers are not in competition but complement each other.

\section{Architecture of Neural Networks}

Neural networks are not only different in the ir learning processes but also different in their structures or topology. Bose (1996) has broadly classified neural networks into recurrent (involving feedback) and nonrecurrent (without feedback) ones. In a little more details, Haykin has divided the network architectures into the following three classes:

\section{Feed-forward Networks}

Feed-forward ANNs allow signals to travel one way only; from input to output. There is no feedback (loops) i.e. the output of any layer does not affect that same layer. Feed-forward ANNs tend to be straight forward networks that associate inputs with outputs. They are extensively used in pattern recognition. This type of organization is also referred to as bottom-up or top-down. Single-layer perceptrons and Multi-layer perceptrons are classes of feed forward networks.

\section{Single-layer perceptrons (feed forward networks)}

The single-layer perceptrons was among the first and simplest learning machines that are trainable. In Haykin's book (1999), perceptron denotes the class of two-layer feed forward networks, 
1) whose first-layer units have fixed function with fixed connection weights from the inputs, and 2) whose connection weights linking this first layer to the second layer of outputs are learnable.

\section{Multi-layer perceptrons (feed forward networks)}

Multi-layer feed forward structures are characterized by directed layered graphs and are the generalization of those earlier single layer structures (Bose, 1996).

\section{Structure and features of MLP}

Multi-layer perceptron (MLP) networks are feed forward nets with one of more layers of nodes between the input and output nodes. The structure of an unadorned multilayer perception network is shown in Figure 1.

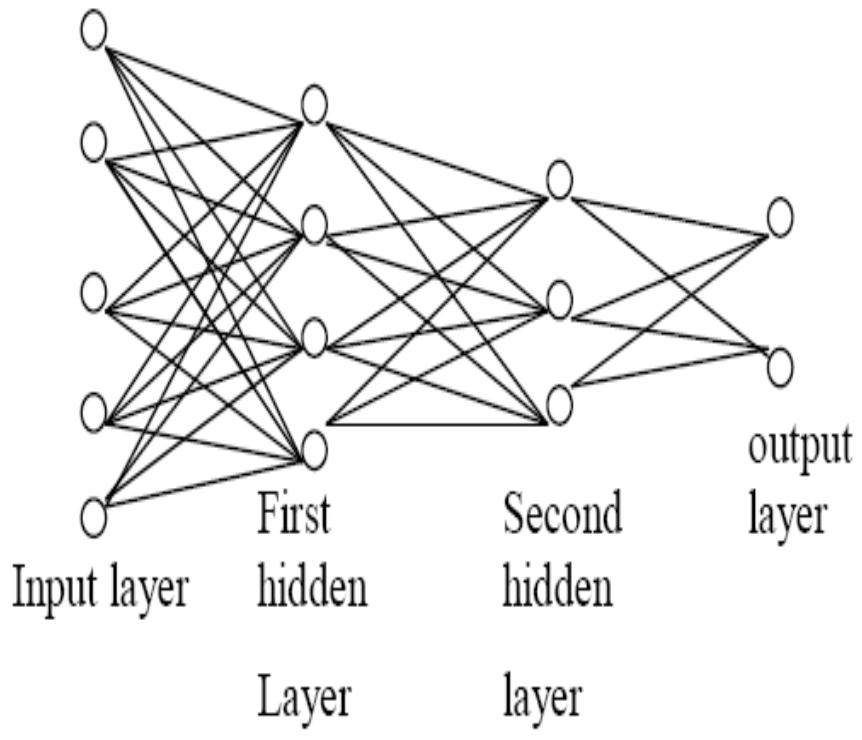

Figure 1. Feed forward multi-layer pe rceptron architecture (Pandya \& Macy, 1996, p.74)

The capabilities of multi-layer perception stem from the nonlinearities used within nodes.

\section{Feedback networks}

Feedback networks (Figure 1) can have signals traveling in both directions by introducing loops in the network. Feedback architectures are also referred to as interactive or recurrent, although the latter term is often used to denote feedback connections in single-layer organizations. In the neural network literature, neural networks with one or more feedback loops are referred to as recurrent networks. A recurrent network distinguishes itself from a feed forward neural network in that it has at least one feedback loop. 


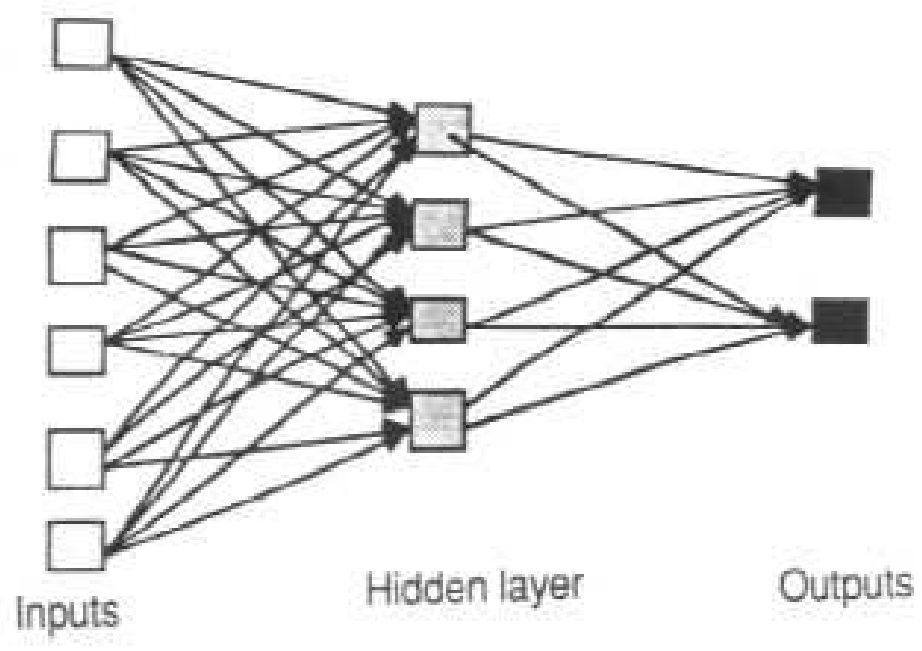

Figure 2. An example of a simple feedforward network

(Stergiou \& Siganos, 1996)

\section{Network Layers}

The commonest type of artificial neural network consists of three groups, or layers, of units: a layer of "input" units is connected to a layer of "hidden" units, which is connected to a layer of "output" units.

The activity of the input units represents the raw information that is fed into the network.

The activity of each hidden unit is determined by the activities of the input units and the weights on the connections between the input and the hidden units.

The behavior of the output units depends on the activity of the hidden units and the weights between the hidden and output units.

\section{Training a Neural Network}

For the most part, a network is trained by changing the weights of the connections between nodes. These weights can be randomly chosen or individually chosen. Usually, a computer program randomly generates values for connection weights. Then, the network is given an input, and it is allowed to process the information through its nodes to produce an output.

\section{The learning process}

The memorization of patterns and the subsequent response of the network can be categorized into two general paradigms:

Associative mapping in which the network learns to produce a particular pattern on the set of input units whenever another particular pattern is applied on the set of input units.

Auto-association: an input pattern is associated with itself and the states of input and output units coincide.

All learning methods used for adaptive neural networks can be classified into two major categories: 
Supervised learning which incorporates an external teacher, so that each output unit is told what its desired response to input signals ought to be.

Unsupervised learning uses no external teacher and is based upon only local information. It is also referred to as self-organization, in the sense that it self-organizes data presented to the network and detects their emergent collective properties.

\section{The back-propagation algorithm}

In order to train a neural network to perform some task, we must adjust the weights of each unit in such a way that the error between the desired output and the actual output is reduced. This process requires that the neural network compute the error derivative of the weights (EW). In other words, it must calculate how the error changes as each weight is increased or decreased slightly. The back propagation algorithm is the most widely used method for determining the EW.

\section{Applications of Neural Networks}

\section{A Simple Neuron}

An artificial neuron is a device with many inputs and one output (Figure 3). The neuron has two modes of operation; the training mode and the using mode. In the training mode, the neuron can be trained to fire (or not), for particular input patterns. In the using mode, when a taught input pattern is detected at the input, its associated output becomes the current output. If the input pattern does not belong in the taught list of input patterns, the firing rule is used to determine whether to fire or not.

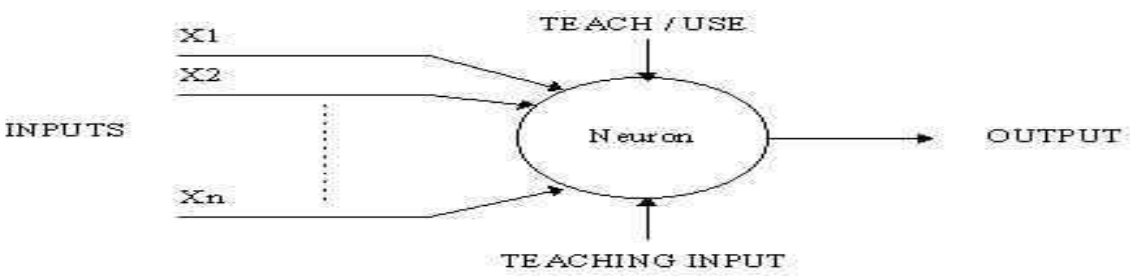

Figure 3. A simple neuron

\section{Firing Rules}

The firing rule is an important concept in neural networks and accounts for their high flexibility. A firing rule determines how one calculates whether a neuron should fire for any input pattern. It relates to all the input patterns, not only the ones on which the node was trained. The firing rule gives the neuron a sense of similarity and enables it to respond 'sensibly' to patterns not seen during training.

\section{Neural Networks in Practice}

Neural networks have broad applicability to real world bus iness problems. In fact, they have already been successfully applied in many industries. Since neural networks are best at identifying patterns or trends in data, they are well suited for prediction or forecasting needs including:

- sales forecasting,

- industrial process control

- customer research 
- data validation

- Risk management.

ANN are also used in the following specific paradigms : recognition of speakers in communications; diagnos is of hepatitis; undersea mine detection; texture analysis; three-dimensional object recognition; hand-written word recognition; and facial recognition.

Typical applications of hardware NNWs are:

\section{OCR (Optical Character Recognition)}

Here, NN was employed in OCR by Caere Incorporation which recorded about 3million dollars prof it on 55million dollar revenue in 1997. The Adaptive Solutions ImageLink OCR Subsystem captures the special high performance hardware required for high throughput. These days a purchase of a new scanner typically includes a commercial OCR program. Ligature Ltd also has an OCR-on-a-Chip example which illustrates a cheap dedicated chip for consumer products

Data Mining: A company named HNC made about 23million dollars profit on 110 million dollar revenue in 1997, on their product called falcon. "Falcon is a neural network based system that examines transaction, cardholder, and merchant data to detect a wide range of credit card fraud..."

\section{Voice Recognition}

Examples are the Sensory Inc. RSC Microcontrollers and ASSP speech recognition specific chips.

Traffic Monitoring: an example is the Nestor TrafficVision Systems.

High Energy Physics: An example is an online data filter built by a group at the Max Planck Institute for the H11 electron-proton collider experiment in the Hamburg using Adaptive Solutions CNAPS boards.

However, most NNW applications today are still run with the conventional software simulation on PC's and workstations with no special hardware add-ons.

\section{Neural Networks in Control Engineering}

The ever-increasing technological demands of our modern society require innovative approaches to highly demanding control problems. Artificial neural networks with their massive paralle lism and learning capabilities offer the promise of better solutions, at least to some problems. By now, the control community has heard of neural networks and wonders if these networks can be used to provide better control solutions to old problems or perhaps solutions to control problems that have withstood our best efforts.

\section{Control system applications}

Neural networks have been applied very successfully in the identification and control of dynamic systems. The universal approximation capabilities of the multilayer perceptron have made it a popular choice for modeling nonlinear systems and for implementing general-purpose nonlinear controllers.

For the purposes of this work we will look at neural networks as function approximators. As shown in Figure 4, we have some unknown function that we wish to approximate. We want to adjust the parameters of the network so that it will produce the same response as the unknown function, if the same input is applied to both systems. For our applications, the unknown function may correspond to a system we are trying to control, in which case the neural network will be the 
identified plant model. The unknown function could also represent the inverse of a system we are trying to control, in which case the neural network can be used to implement the controller.

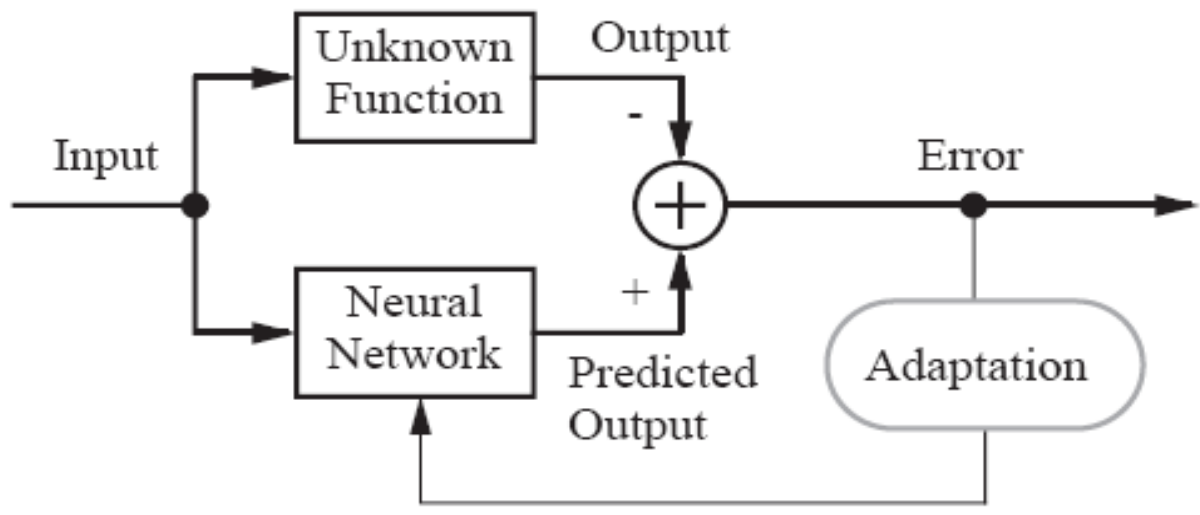

Neural Network as Function Approximator

Figure 4. Neural Network as Function Approximator (Hagan, Demuth, \& De Jesús, 2002)

\section{Fixed Stabilizing Controllers}

Fixed stabilizing controllers (see Figure 5) have been proposed in (Kawato, 1990). This scheme has been applied to the control of robot arm trajectory, where a proportional controller with gain was used as the stabilizing feedback controller. We can see that the total input that enters the plant is the sum of the feedback control signal and the feedforward control signal, which is calculated from the inverse dynamics model (neural network). That model uses the desired trajectory as the input and the feedback control as an error signal. As the NN training advances, that input will converge to zero. The neural network controller will learn to take over from the feedback controller. The advantage of this architecture is that we can start with a stable system, even though the neural network has not been adequately trained.

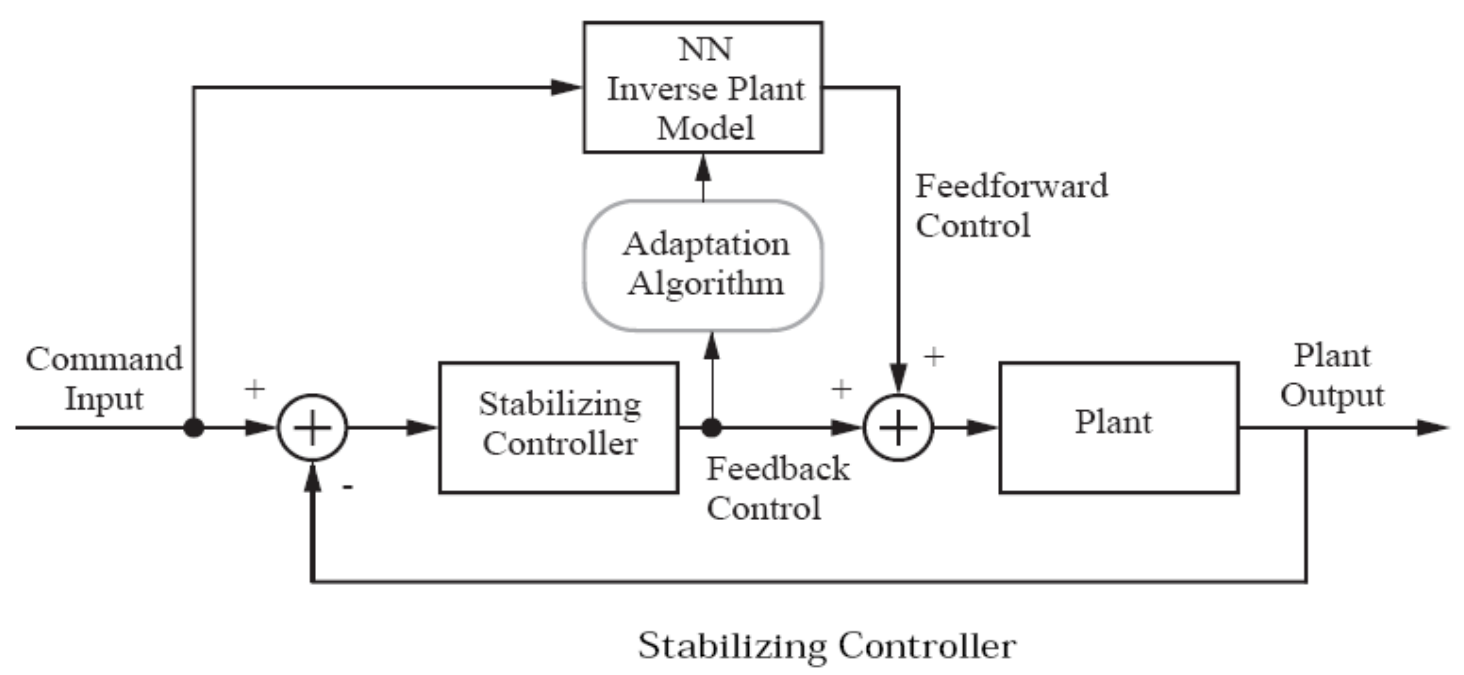

Figure 5. A Stabilizing Controller (Hagan et al., 2002) 
We have selected one type of network, the multilayer perceptron. We have demonstrated the capabilities of this network for function approximation, and have described how it can be trained to approximate specific functions. We then presented control architecture which use neural network function approximators as basic building blocks. Control engineering also involves robotics, where Intelligent Control is the discipline that implements Intelligent Machines(IMs) to perform anthropormorphic tasks with minimum supervision and interaction with a human operator (Jegede, Awodele, Ajayi, \& Ndong, 2007).

\section{Agricultural Control System Engineering}

Control and management of agricultural machinery offers many opportunities for application of general purpose empirical models. The nature of agricultural machines creates the need for modeling systems that are robust, noise tolerant, adaptable for multiple uses, and are extensible. Artific ial Neural Networks (ANNs) have these characteristics and are attractive for use in control and modeling in agricultural machinery.

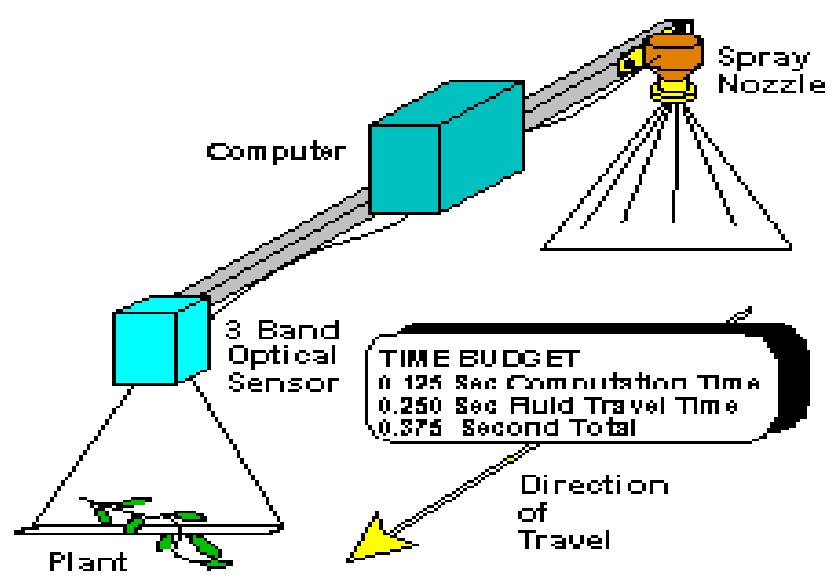

Figure 6. Sprayer Se nsor and Nozzle Element (Zhang, Yang, \& El-Faki, 1994)

\section{Weed Detection in Sprayers}

Figure 6 presents a schematic of the sensor and spray nozzle element component of the sprayer. The complete sprayer consisted of many of the sensor-nozzle elements placed in parallel on a single spray boom.

A sensor was fabricated to detect color on the surface of the ground in a 7.5 by $50-\mathrm{cm}$ wide image. Three color bands; green, red, and near infra-red were sensed. The signals from the sensor were digitized with a $68 \mathrm{HC} 11$ based controller using the on-chip 8-bit A/D converter. The $68 \mathrm{HC} 11$ based computer was also used to activate a solid-state switch that energized a solenoid valve in the spray nozzle. The intent of control in the system was to sense the presence of a weed by color and to activate the nozzle to spray the plant at the point in time that the plant was under the nozzle. A time budget is shown in the figure. If computing time plus the time required for the fluid to reach the ground once it emerges from the nozzle was insignificant, the sensor and nozzle could be located together. Agricultural sprayers based on optical sensing and control of spray nozzle activation currently exists on the market.

\section{Weed identification}

Zhang, Yang, \& El-Faki (1994) reported the use of ANNs to process color images of weeds in a winter-wheat environment with the objective of being able to distinguish between weeds and 
other components of the image. They were particularly interested in detecting weeds with reddish stems.

An ANN was also developed to allow color patterns to be recognized in an agricultural weed sprayer application by Stone (1994).

\section{Neural Networks in Electrical Engineering}

Artificial Neural Network (ANN) is currently a 'hot' research area in electrical engineering. The model used to simulate artificial neural networks is based on the biological nerve cell or neuron shown in Figure 7. Electrical signals arising from impulses from our receptor organs (e.g. eyes, ears) are carried into neurons on dendrites.

\section{Signal classification with Perceptron}

A problem of particular interest to electrical engineers is that of signal detection, particularly in a noisy environment. Methods such as filtering and signal averaging have been used successfully.

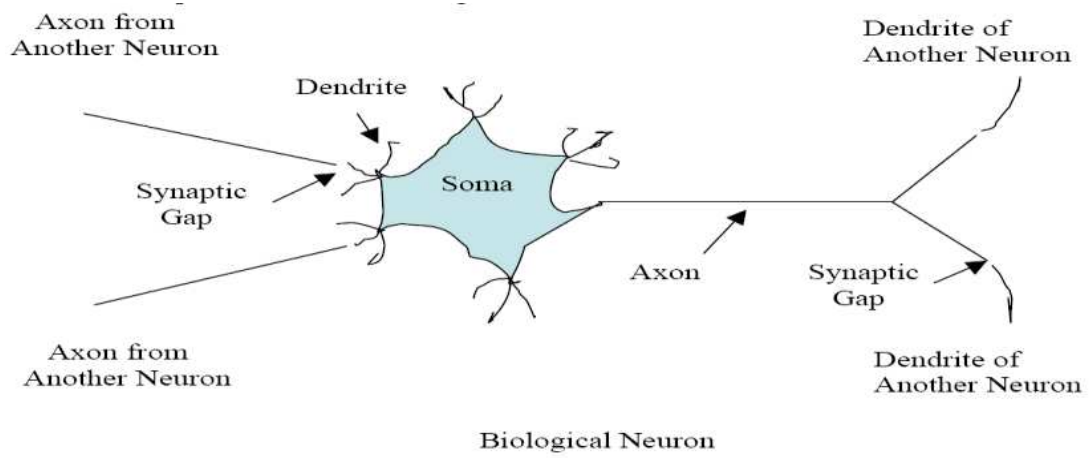

Figure 7. A Biological Neuron (Howard, 2006)

\section{Neural Networks and its application in Civil Engineering}

Neural networks have gained a broad interest in civil engineering problems. They are used as an alternative to statistical and optimization methods as well as in combination with numerical simulation systems. Application areas in Civil Engineering are e.g. forecasting, water management, control and decision support systems.

\section{Limitations of Neural Networks}

The major issues of concern today are the scalability problem, testing, verification, and integration of neural network systems into the modern environment. Neural network programs sometimes become unstable when applied to larger problems. The defence, nuclear and space industries are concerned about the issue of testing and verification. The mathematical theories used to guarantee the performance of an applied neural network are still under development. The solution for the time being may be to train and test these intelligent systems much as we do for humans. Also there are some more practical problems like: the operational problem encountered when attempting to simulate the parallelis $m$ of neural networks instability to explain any results that they obtain. Networks function as "black boxes" whose rules of operation are completely unknown. 
Likew ise, in OCR, we find that one cannot claim Neural Networks (NNWs) are conquering the world, because one does not feed the pixels of the picture file into a single giant NNW and out pops the text. To turn a picture of text into a text file, a dozen or more steps must be completed successfully by the OCR program. For example, an OCR system might follow the step in the diagram in Figure 8.

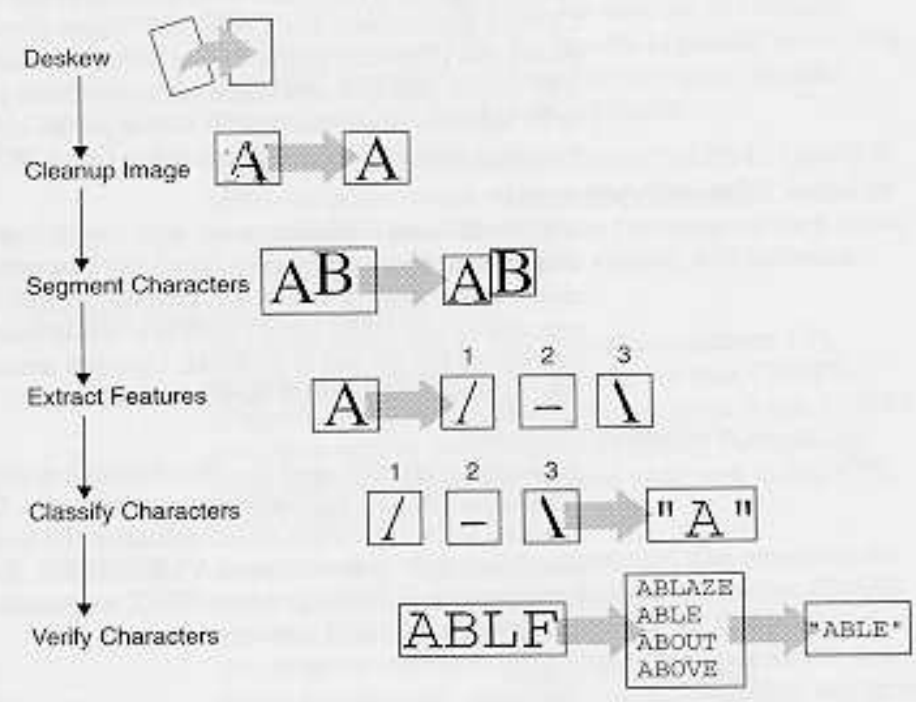

Figure 8. (From Adaptive Solutions CNAPS User Guide).

Note that Adaptive Solution CNAPS is an example of a general, but expensive system that can be reprogrammed for many kinds of tasks.

Designers of OCR programs may choose NNWs to accomplish one or more of these steps with NNWs while using for other steps other techniques such as conventional AI (If-Then rules), statistical models, hidden Markov models, etc. The point is that NNWs are becoming commonly used tools but, just like other techniques such as Fast Fourier Transform and least squares fit, they are still only tools, not the whole solution. Few real problems of interest can be totally solved by a single NNW. It is also true that implementing NNWs in Hardware and Software to run on them is relative ly expensive.

With the aforementioned, one quickly begins to see why the business of Neural Network hardware has not boomed the way some in the field expected back in the 1980's.

\section{Conclusion}

Prediction for the future rests on some sort of evidence or established trend which, with extrapolation, clearly takes us into a new realm. Neural Networks will fascinate user-specific systems for education, information processing, entertainment, genetic engineering, neurology and psychology.

Programs could be developed which require feedback from the user in order to be effective but simple and "passive" sensors (e.g. fingertip sensors, gloves, or wristbands to sense pulse, blood pressure, skin ionization, and so on), could provide effective feedback into a neural control system. NN's ability to learn by example makes them very flexible and powerful. Perhaps the most 
exciting aspect of neural networks is the possibility that some day 'conscious' networks might be produced.

\section{Recent advances and future applications of NNs include:}

\section{Integration of fuzzy logic into neural networks}

Fuzzy logic is a type of logic that recognizes more than simple true and false values, hence better simulating the real world. For example, the statement today is sunny might be $100 \%$ true if there are no clouds, $80 \%$ true if there are a few clouds, $50 \%$ true if it's hazy, and $0 \%$ true if rains all day. Hence, it takes into account concepts like -usually, somewhat, and sometimes.

Fuzzy logic and neural networks have been integrated for uses as diverse as automotive engineering, applicant screening for jobs, the control of a crane, and the monitoring of glaucoma.

\section{Pulsed neural networks}

"Most practical applications of artificial neural networks are based on a computational model involving the propagation of continuous variables from one processing unit to the next. In recent years, data from neurobiological experiments have made it increasingly clear that biological neural networks, which communicate through pulses, use the timing of the pulses to transmit information and perform computation. This realization has stimulated significant research on pulsed neural networks, including theoretical ana lyses and model development, neurobiological modeling, and hardware implementation" (From http://www.tu-graz.ac.at/igi/maass/PNN.html).

\section{Hardware specialized for neural networks}

Some networks have been hardcoded into chips or analog devices? This technology will become more useful as the networks we use become more complex.

The primary benefit of directly encoding neural networks onto chips or specialized analog devices is SPEED!

NN hardware currently runs in a few niche areas, such as those areas where very high performance is required (e.g. high energy physics) and in embedded applications of simple, hardwired networks (e.g. voice recognition).

Many NNs today use less than 100 neurons and only need occasional training. In these situations, software simulation is usually found sufficient

When NN algorithms develop to the point where useful things can be done with 1000's of neurons and 10000's of synapses, high performance NN hardware will become essential for practical operation (From http://www.particle.kth.se/ lindsey/HardwareNNWCourse/).

\section{Improvement of existing technologies}

All current $\mathrm{NN}$ technologies will most likely be vastly improved upon in the future. Everything from handwriting and speech recognition to stock market prediction will become more sophisticated as researchers develop better training methods and network architectures.

NNs might, in the future, allow:

- robots that can see, feel, and predict the world around them

- improved stock prediction

- common usage of self-driving cars

- composition of music 
- handwritten documents to be automatically transformed into formatted word processing documents

- trends found in the human genome to aid in the understanding of the data compiled by the Human Genome Project

- Self-diagnosis of medical problems using neural networks and much more!

In conclusion it should be stated that even though neural networks have a huge potential, we will only get the best of them when they are integrated with computing, AI, fuzzy logic and related subjects.

\section{References}

Bose, N. K.; \& Liang P. (1996). Neural network fundamentals with graphs, algorithms, and applications. McGraw-Hill.

DARPA Neural Network Study. (1988). AFCEA International Press, p. 60)

Hagan, M. T., Demuth, H. B. \& De Jesús, O. (2002). An introduction to the use of neural networks in control systems. International Journal of Robust and Nonlinear Control, 12(11,) 959- 985.

Haykin, S. (1999). Neural networks: A comprehensive foundation (2nd ed.). Upper Saddle River, New Jersey: Prentice Hall.

Howard S. (2006). Neural networks in electrical engineering. Proceedings of the ASEE New England Section 2006 Annual Conference (Session 1A - Electrical \& Computer Engineering).

Jegede, O., Awodele, O., Ajayi, A., \& Ndong M. (2007). Develop ment of a microcontroller based robotic arm. Proceedings of the 2007 CSITEd Conference" (Information Technology). Retrieved from http://csited.org/2007/340lawCSITEd.pdf

Kawato M. (1990). Computational schemes and neural network models for formation and control of mu ltijoint arm trajectory. In T. Miller, R. S. Sutton, \& P. J. Werbos (Eds.), Neural networks for control (pp.197-228). Cambridge, MA: MIT Press.

Pandya, A. S., \& Macy, R. B. (1996). Pattern recognition with neural networks in C++. CRC Press.

Stergiou, C., \& Siganos, D. (1996). Neural networks. Retrieved from http://www.doc.ic.ac.uk/ nd/surprise 96/journal/vol4/cs 11/report.html

Stone, M. L. (1994). Embedded neural networks in real time controls. SAE Paper 941067. 45th Annual Earthmoving Industry Conference. SAE, Warrendale PA.

Zhang, N., Yang, Y., \& El-Faki, M. (1994). Neural-network application in weed identification using color digital images. ASAE Paper No. 94-3511.

\section{Biographies}

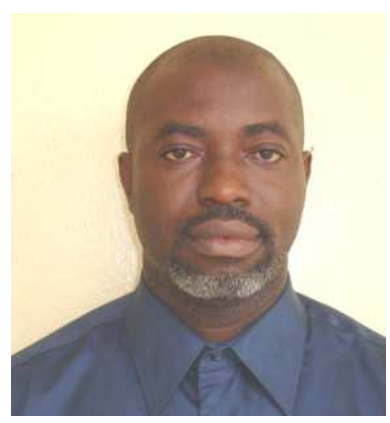

Oludele Awodele holds a Ph.D in Computer Science from the University of Agriculture, Abeokuta, Nigeria. He has several years experience of teaching computer science courses at the university level. He is currently a lecturer in the department of Computer Science and Maths, Babcock University, Nigeria. He is a full member of the Nigeria Computer Society and the Computer Professional Registration Council of Nigeria. His areas of interest are Artificial Intelligence and Computer Architecture. He has published works in several journals of international repute. 


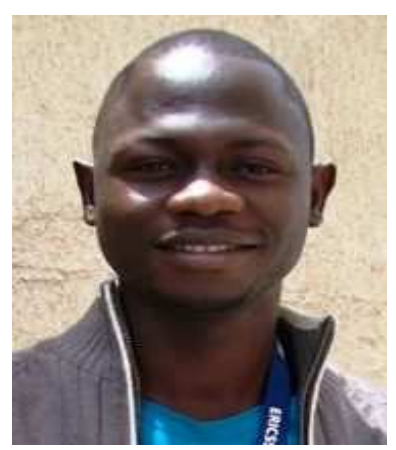

Olawale Jegede works with LM Eric sson Nigeria (Core Network Engineer: MPBN:IP/MPLS). He is a graduate of Babcock University, Nigeria where he obtained a first class degree in Computer Engineering Technology. He is a Cisco Certified Network Professional. He is a member of the Nigeria Society of Engineers and the Nigeria Computer Society. His areas of interest include: Telecommunications and Networking, Artificial Intelligence, Digital Electronics, and Control. He has published works in several journals of international repute. 